\title{
The configural advantage in object change detection persists across depth rotation
}

\author{
SIMONE K. FAVELLE \\ University of Wollongong, Wollongong, New South Wales, Australia \\ WILLIAM G. HAYWARD \\ University of Hong Kong, Hong Kong \\ DARREN BURKE \\ Macquarie University, Sydney, New South Wales, Australia \\ and \\ STEPHEN PALMISANO \\ University of Wollongong, Wollongong, New South Wales, Australia
}

\begin{abstract}
Although traditionally there has been a debate over whether object recognition involves 3-D structural descriptions or 2-D views, most current approaches to object recognition include the representation of object structure in some form. An advantage for the processing of structural or configural information in objects has been recently demonstrated using a change detection task (Keane, Hayward, \& Burke, 2003). We report two experiments that extend this finding and show that configural information dominates change detection performance regardless of an object's orientation. Experiment 1 demonstrated the advantage that configural information has over shape and part arrangement information in change detection across four different object rotations in depth. Experiment 2 showed that this advantage occurs for both categorical and coordinate configural changes. These results are consistent with the hypothesis that configural information is a critical feature of object representations and that this information is utilized effectively in object recognition across changes in viewpoint.
\end{abstract}

As people move around the world and the world moves around people, the distance, position, view, shape, and shading of objects continually change. Despite potentially substantial variations in the retinal images produced by objects, the ability of the human brain to recognize them is not disrupted. What information in the visual environment is being exploited that allows the brain to recognize objects as being the same or to discriminate between different objects? For the most part, early theories about visual object recognition and the problem of recognizing 3-D objects (despite 2-D retinal image input) fell into one of two classes of models. The proponents of one class of models postulated the existence of viewpoint-invariant representations of objects that included 3-D information about parts and spatial relations. Those on the side of the other class argued that representations were based on 2-D images and were viewpoint dependent. As disparate as these two classes may appear, recent research in this area

This research was partially supported by Grant CUHK4232/02H from the Hong Kong Research Grants Council. We thank Alex Lee for help with the analyses. We also thank the two anonymous reviewers for their helpful comments in relation to earlier drafts of the manuscript. Correspondence concerning this article should be addressed to S. K. Favelle, Department of Psychology, University of Wollongong, Northfields Ave., Wollongong 2522, NSW, Australia (e-mail: simone_favelle@uow.edu.au). has begun to investigate the cooperation of these models (Foster \& Gilson, 2002).

One particular object property that has been posited as a key factor in the cooperation of viewpoint-dependent and viewpoint-invariant theories is structure, or spatial configuration (Edelman \& Intrator, 2000; Foster \& Gilson, 2002; Hummel \& Stankiewicz, 1998; Tarr \& Bülthoff, 1998). Tarr and Bülthoff were among the first researchers to suggest that structural information might be encoded along with multiple 2-D viewpoint-dependent representations of objects. Subsequently, models of object representation and recognition have begun to openly incorporate structure and spatial relations with 2-D view information. Edelman and Intrator $(2000,2001,2003)$, for example, proposed the chorus of fragments (CoF) theory of object representation, which posits that structure is central to object representation and that the constituent parts of an object are coarsely coded 2-D shape fragments, not volumetric primitives. A recent model of object recognition proposed by Foster and Gilson (2002) also effectively combines both structural and view-based information. Foster and Gilson's model is based on empirical data that show sensitivity to both structural and 2-D view information. They found that although differences in object structure were discriminated more readily than metric property differences, object structure and metric properties displayed very similar viewpoint dependence. On the basis of their results, Foster and Gilson 
argued for a simple additive model of object recognition that includes independent terms for object structure and 2-D view information.

Object structure or configuration has been widely investigated in relatively simple objects and has been generally shown to be an important type of information in visual processing. For example, the dominance of configural information over component properties has been demonstrated in the perceptual organization of 2-D objects (see Kimchi, 2003, for a review). An advantage for configural information has also been demonstrated in change detection studies with novel 3-D objects (Favelle, Palmisano, Burke, \& Hayward, 2006; Keane, Hayward, \& Burke, 2003). Keane et al. investigated change detection in novel objects for (1) the configuration of object parts, (2) the shape of those parts, and (3) the relative arrangement of those parts. Using a one-shot change detection task, Keane et al. found that observers detected changes to the configuration of object parts more quickly and more accurately than they did changes to the shape of the parts or a switching of the parts. This result suggests that information regarding the global configuration of parts is better encoded than are more local details, such as part shape. Furthermore, categorical configural changes were found to be easier to detect than coordinate (metric-based) configural changes. Thus, it is the configural nature of the change and not the size of the change per se that is responsible for this configural advantage, in which changes to the configuration of object parts are more quickly and more accurately detected than are changes to the shape of those parts or a switching of those parts.

The above-mentioned configural effects were found in conditions in which viewpoint was held constant. However, objects are rarely seen from a single viewpoint. Our visual experience typically includes numerous different views of objects in the environment. The aim of the present article is to further investigate the configural advantage finding. In particular, we wish to investigate whether these differences in the detection of changes in (1) configuration, (2) switching, and (3) changing of object parts remain when viewpoint changes and, consequently, whether this configural advantage can be used to add to what we know about object perception and to models that combine configural or structural information with 2-D view information.

Evidence exists that spatial configuration may not be critical for object recognition across changes in viewpoint. Johnston and Hayes (2000) examined recognition for objects that were discriminable based on either morphological (shape) information about parts or spatial configuration of parts across various viewpoint manipulations (stimuli could be rotated either in depth or in the picture plane). In four experiments, using both sequential matching and learning/recognition tasks, performance for objects that were discriminable on the basis of the spatial configuration of parts was poorer than it was for objects that were discriminable by the shape of their parts, particularly for delayed recognition. Furthermore, they found a larger viewpoint effect for spatial configu- ration changes than for part shape changes; in effect, this means that the differences between the conditions became larger as the objects were rotated from the studied view. Johnston and Hayes argued that the reason for this poor performance is that 2-D projections of relative positions of parts are highly viewpoint dependent and do not allow for accurate discrimination between objects under depth rotation.

The shape and configuration change conditions used by Keane et al. (2003) correspond approximately to Johnston and Hayes's (2000) morphological and spatial relations object sets. Both have one condition in which part shape is manipulated while overall spatial relations among parts are held constant, and another condition in which part identity or shape remains constant while spatial configuration is manipulated. However, the results of the two studies were very different. Whereas Keane et al. found better performance when spatial relations were manipulated, Johnston and Hayes reported better performance when object shape was manipulated.

One obvious difference between the studies is that Johnston and Hayes (2000) included a viewpoint manipulation, whereas Keane et al. (2003) always showed objects from a single viewpoint. Thus, Keane et al.'s finding that spatial configuration changes were detected more easily may be due to the fact that they only examined one viewpoint. A possible integration of the two sets of results would be the prediction that part shape should become more useful to the observer as the viewpoint difference between the two stimuli increases. However, if object representation and recognition is accurately modeled by the combination of structural and view-based information, then the configural effects found at $0^{\circ}$ rotation should persist across changes in viewpoint.

Another difference between the studies carried out by Keane et al. (2003) and Johnston and Hayes (2000) lies in the experimental tasks used. Johnston and Hayes used both sequential matching and delayed learning tasks to tap into short- and long-term object representations, respectively. They found the pattern of performance to be fairly similar between these tasks. The experimental task employed by Keane et al. (and by the authors of the present article) was a one-shot change detection task with a same-different response. In essence, both studies used a sequential matching task (typically used to investigate short-term object recognition; see, e.g., Hayward \& Tarr, 1997; Johnston \& Hayes, 2000; Tarr, Bülthoff, Zabinski, \& Blanz, 1997), but the analysis pertained primarily to the different rather than the same trials. The rationale behind the use of change detection is that by discovering the types of changes to objects that are easily detected we could identify object properties that are exploited and represented by the visual system.

In the two experiments in this article, we investigated whether detection performance for object property changes is differentially affected by changes to an object's orientation in depth. If the configural advantage found previously for object change detection (Keane et al., 2003) generalizes to new viewpoints, that would suggest 
that configural information is readily utilized across the different viewpoints and would lend support to theories of object representation and recognition that propose a key role for object structure. In Experiment 1, we investigated the detection of three types of property changes to objects across rotations in depth. In Experiment 2, we performed a more systematic investigation of the use of configural information across viewpoints by using separate changes to categorical and coordinate relations.

\section{EXPERIMENT 1}

The object properties investigated in Experiment 1 were the same as those analyzed by Keane et al. (2003). The changes to be detected were (1) configural, in which the location of a part on the object body would change, resulting in a new part configuration; (2) shape based, in which the shape of one part would change; or (3) a switch, in which the locations of two parts would switch (see Figure 1A). Change detection performance was measured across changes in object orientation in depth, from $0^{\circ}$ to $45^{\circ}$. If changes to configural information were to be detected more quickly and accurately than were shape or switch changes, regardless of an object's orientation, then this would suggest that object structure is important information that is retained across viewpoint changes.

\section{Method}

Subjects. Thirty undergraduate students participated and were tested individually, and they received course credit for participating.

Materials. The stimuli were rendered images of 3-D novel objects. Each object was composed of a main body with three appendage parts. The appendages were attached to the body at three of six possible positions. On each of the six "standard" objects, there were changes in part configuration and part shape and a switch of appendage parts. The objects were shown rotated by $0^{\circ}, 15^{\circ}, 30^{\circ}$, and $45^{\circ}$ around the vertical axis, for a total of 240 different object exemplars (see Figure 1B). The mask used in Experiment 1 was $400 \times$ 300 pixels in size and consisted of elements from a variety of object images. The experiment was controlled by RSVP software (www .tarrlab.org) on Macintosh computers with a 17-in. Macintosh CRT $(640 \times 480$ pixels $)$ monitor.

Procedure. The experiment consisted of 360 randomly ordered trials. Each trial began with a fixation cross appearing for $500 \mathrm{msec}$ at the center of the screen, followed by the first object for $2 \mathrm{sec}$, a mask for 1,500 msec, and finally the second object, which remained on the screen until the subject responded. The responses for each trial timed out after $5 \mathrm{sec}$. The next trial began $1 \mathrm{sec}$ after the subject made a response or the trial timed out. Each stimulus was jittered by 25 pixels - that is, it was randomly placed at a position 25 pixels in any direction from the center of the screen.

\section{(A)}

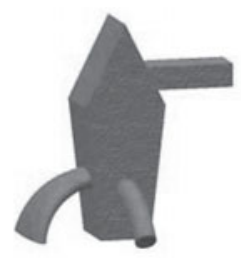

Standard Object
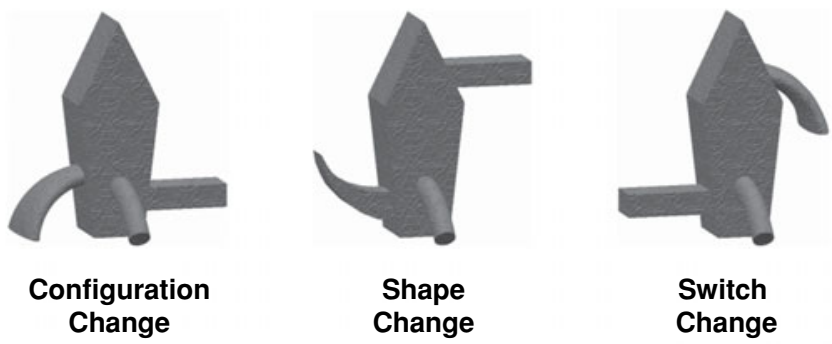

(B)

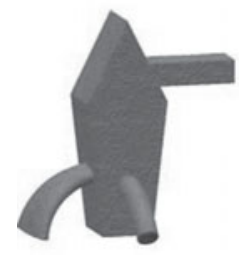

$0^{\circ}$

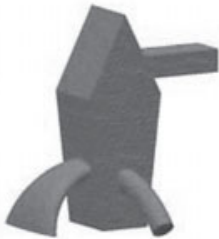

$15^{\circ}$

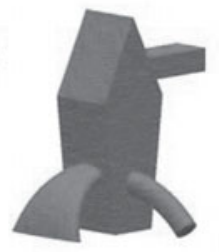

$30^{\circ}$

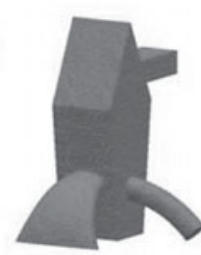

$45^{\circ}$

Figure 1. Example of (A) the three different types of change (configuration, shape, and switch) used in Experiment 1 and (B) one of the object stimuli rotated $45^{\circ}$ around the vertical axis. 
Half the trials were same trials, and the other half were different. The different trials were split equally into the three change conditions. The second object was presented at a viewpoint of $0^{\circ}, 15^{\circ}, 30^{\circ}$, or $45^{\circ}$ rotated in depth. Furthermore, the second object was either identical to the first or different in one of three ways: (1) part shape, (2) a switching of parts, or (3) spatial configuration. The subjects were instructed to indicate, regardless of differences in rotation, whether the two objects presented to them were the same or different by pressing corresponding keys on a keyboard.

\section{Results and Discussion}

Accuracy (proportion correct), sensitivity $\left(d^{\prime}\right)$, and reaction time (RT) data were collected. The $d^{\prime}$ data measured the observers' sensitivity to the different types of change independently of their response bias (Macmillan \& Creelman, 1991). Each observer's same-different responses were converted into hit rates $(\mathrm{H})$ and false alarm rates $(\mathrm{F})$. These rates, expressed as probabilities ranging from 0 to 1 , were used to calculate $d^{\prime}$ as follows:

$$
d^{\prime}=2 z\left[0.5\left\{1+\left[2 p(\mathrm{c})_{\max }-1\right]^{0.5}\right\}\right],
$$

where $p(\mathrm{c})_{\max }=\Phi\{[z(\mathrm{H})-z(\mathrm{~F})] / 2\}$ (Macmillan \& Creelman, 1991, chapter 6). RT data analysis was conducted using accurate responses. Accuracy, $d^{\prime}$ and RT data were analyzed using a $3 \times 4$ repeated measures ANOVA including the within-subjects factors of change type (configuration, shape, or switch) and orientation $\left(0^{\circ}, 15^{\circ}, 30^{\circ}\right.$, or $45^{\circ}$ ).

Considering proportion correct (see Figure 2), subjects were more accurate at detecting a spatial configuration change (.89) and less accurate at detecting either a switch (.81) or a shape change $(.81)$. A $3 \times 4$ repeated measures ANOVA for accuracy showed significant main effects for change type $[F(2,58)=13.05, p<.01]$ and orientation $[F(2,58)=11.07, p<.01]$ but no interaction between change type and orientation $[F(6,174)=1.71, p=.12]$. Bonferroni-adjusted post hoc contrasts showed that configuration changes were detected significantly more accurately than were either shape or switch changes (both $p s<.01)$ and that there was no significant difference in accuracy between shape and switch changes $(p=.73)$. A trend analysis was conducted for the main effect of orientation on accuracy. The linear and quadratic contrasts were significant $[F(1,29)=25.38, p<.01$, and $F(1,29)=$ $7.47, p<.01$, respectively]. The cubic contrast was not significant $(p=.56)$. The quadratic trend was interpreted as indicating that there was very little difference in detection performance between the $0^{\circ}$ and $15^{\circ}$ conditions. As seen in Figure 2, the $d^{\prime}$ data show the same pattern as the accuracy data. Both the main effects of change type and orientation were significant, whereas their two-way interaction was not significant, indicating that our experimental manipulations altered subjects' sensitivity to change, rather then their response bias.

The RT data reflect the pattern found in both the accuracy and the $d^{\prime}$ data (see Figure 3 ). A $3 \times 4$ repeated measures ANOVA on RT also showed significant main effects for change type $[F(2,58)=11.57, p<.01]$ and orientation $[F(2,58)=13.32, p<.01]$, with no interac- tion between change type and orientation $[F(6,174)=$ $1.17, p=.32]$. Bonferroni-adjusted post hoc contrasts showed that configuration changes were detected significantly more quickly than were either shape or switch changes (both $p \mathrm{~s}<.01$ ) and that there was no significant difference in RT between shape and switch changes ( $p=$ .15). A trend analysis was conducted for the main effect of orientation on RT. The linear contrast was significant $[F(1,29)=36.10, p<.01]$. Neither the quadratic nor the cubic contrast was significant (both $p \mathrm{~s}>.06$ ).

Overall, detection of change to 3-D objects was found to be viewpoint dependent (Johnston \& Hayes, 2000; Tarr, Williams, Hayward, \& Gauthier, 1998). However, contrary to Johnston and Hayes's findings, the present results show that accuracy and sensitivity are greater and RT is faster in the detection of configural changes than of either shape or switch changes. There was no significant difference between the shape and switch conditions in accuracy, $d^{\prime}$, or RT. This suggests that coarse configural information was utilized quickly and accurately across different object viewpoints in depth and that observers were more sensitive to changes in this information than to changes in information about the shape of individual components.

Magnitude of change. When we compare different types of change, a concern arises regarding the quantitative differences in the magnitude of the changes. This issue was addressed in a previous article (Keane et al., 2003), in which the detection of the same kinds of changes (configural, shape, and switch) made to novel objects was investigated. In that article, objects were always viewed at $0^{\circ}$ rotation. Keane et al. employed a quantitative measure of change in which the number of pixels that changed from black to white - or vice versa - in two silhouetted objects was calculated (see also Williams \& Simons, 2000). This pixel change measure approximates the extent to which external contours and internal visual features differ between two stimuli. Keane et al. found that this measure could not account for the differences in accuracy between the three types of change, suggesting that it was indeed the type of change, rather than the quantitative nature of the change, that was responsible for their findings.

The present accuracy data were subjected to a similar analysis. We employed two different measures of quantitative change (see the Appendix for the mean values for each measure in each condition). The first was the pixel change measure that was used previously by Keane et al. (2003) and Williams and Simons (2000). The second was a measure of change in the eight-bit (256-color) images. ${ }^{1}$ This method gives a quantitative measure of color change. Although this method is somewhat limited (since similarities in the color palette table may not equate with psychological reality), it allows us to differentiate between subtle and large changes in color. ${ }^{2}$ Two separate ANCOVAs were conducted, both with proportion correct as the dependent value and either pixel change or change in color image as the covariate. The results of both ANCOVAs showed that neither the pixel change nor the change in color image covariate significantly predicted accuracy $[F(1,3)=0.16$, $p=.72$, and $F(1,3)=0.07, p=.81$, respectively]. Thus, 
A

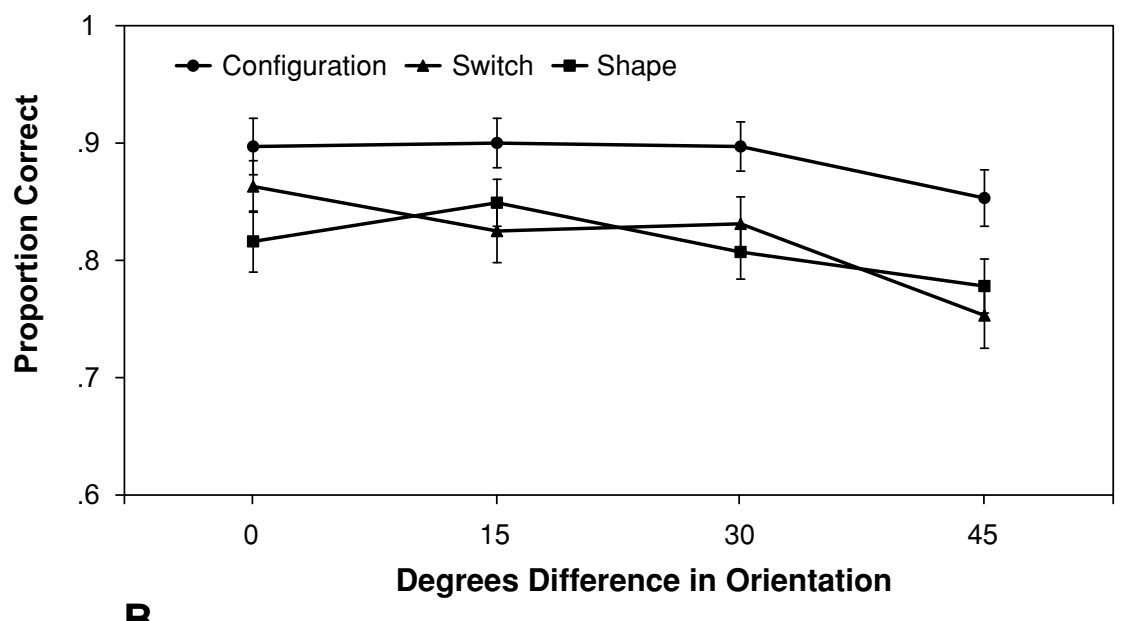

B

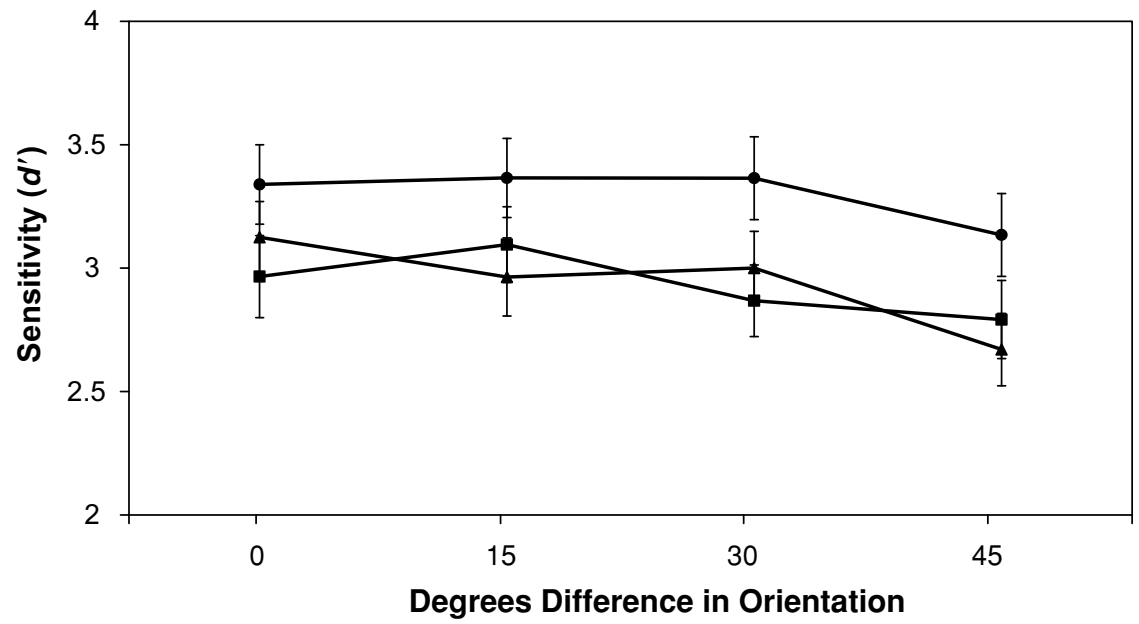

Figure 2. Mean accuracy (A) and sensitivity to change (B) on the change detection task in Experiment 1 as a function of change type and object orientation. Error bars represent $\pm S E$.

although there were physical differences in configuration, switch, and shape changes, these were clearly not large enough to account for the configural advantage found here and in previous studies.

\section{EXPERIMENT 2}

In Experiment 1, changes to part configuration were consistently detected more quickly and more accurately than were part shape and switch changes - even across object rotations in depth. Thus, information about the spatial relations between components seems to be encoded in a fashion that can be deployed not just for images depicting the object from the same viewpoint (Keane et al., 2003) but also for images depicting the object across different viewpoints. If this is indeed the case, in what form is this information encoded? Different models make distinct predictions. Structural description theories of object recognition propose that object parts are represented in terms of categorical spatial relations (e.g., one part is above or below another; see Biederman, 1987; Hummel \& Stankiewicz, 1996). Alternatively, viewpoint-dependent theories rely on coordinate spatial relations (e.g., the metric direction and distance between two points; see Bülthoff, Edelman, \& Tarr, 1995).

When viewpoint was held constant, Keane et al. (2003) found that change detection for categorical relations was more accurate than for coordinate relations (although RTs were not different), in accordance with structural description theories of object recognition. Given this result, we expected that categorical spatial relations would also be more useful when the studied image had to be generalized across changes in viewpoint. In Experiment 2, we tested subjects' ability to detect changes to categorical and coordinate spatial relations across different object rotations in depth. Importantly, as in Keane et al.'s study, the categorical and coordinate spatial relations changes investigated in Experiment 2 were created so that it was only the rela- 


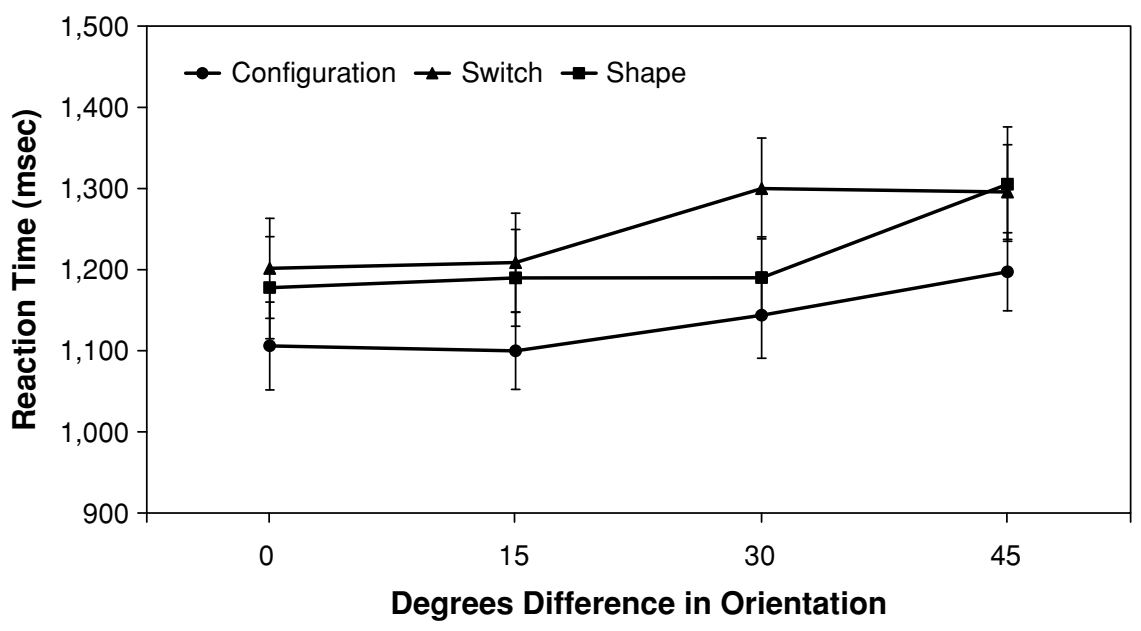

Figure 3. Mean RT on the change detection task in Experiment 1 as a function of change type and object orientation. Error bars represent $\pm S E$.

tions between parts that distinguished the two changes; the quantitative difference between categorical and coordinate changes was identical. We expected to replicate the pattern of results found by Keane et al. at $0^{\circ}$ rotation. The main concern of Experiment 2, however, was whether this pattern would remain across changes in viewpoint. In particular, we were interested in (1) whether we would replicate the general configural detection advantage over switch and shape detection, and (2) whether categorical changes to configuration would be detected more easily than coordinate changes.

\section{Method}

Subjects. Twenty-nine undergraduate students participated and were tested individually, and they received course credit for participating.

Materials. The stimuli that we used in Experiment 2 were a subset of those used by Keane et al. (2003, Experiment 4; see Figure 3 from this earlier article). The stimuli were rendered images of 3-D novel objects that were similar to those we used in Experiment 1. Each object was composed of a main body with three appendage parts. The appendages were attached to the body at three of nine possible positions. There were six "standard" objects, each having categorical, coordinate, or shape changes made to them. For any particular object, the same part was involved in all the experimental conditions. The two other appendage parts were also involved in configuration and shape changes; however, these other changes were included so that the subjects would not focus attention solely on the part involved in the categorical or metric change (which would perhaps bias results). The distance that an appendage part was moved was quantitatively identical for both categorical and coordinate changes. The difference between categorical and coordinate changes involved the spatial relationship between the moved appendage and the two other appendage parts. The categorical change involved one part moving along the body so that it went from above a second appendage to below it (or vice versa), in line with the third appendage. The coordinate change involved one part moving along the body so that it was further above or below the two other appendages (see Figure 4).

Objects were shown at four different viewpoints in depth. Each object was rotated around the vertical axis $0^{\circ}, 15^{\circ}, 30^{\circ}$, and $45^{\circ}$, for a total of 288 different object exemplars. All objects were photore- alistically rendered with the same color and texture and the entire background screen was white. The mask used in this experiment was $425 \times 312$ pixels in area. The experiment was controlled by RSVP software on Macintosh computers with a 17 -in. Macintosh CRT $(640 \times 480$ pixels $)$ monitor.

Procedure. The experiment consisted of 528 randomly ordered trials. The procedure was the same as that used in Experiment 1.

\section{Results and Discussion}

Three separate $3 \times 4$ repeated measures ANOVAs, including the within-subjects factors of change type (categorical configuration, coordinate configuration, or shape) and orientation $\left(0^{\circ}, 15^{\circ}, 30^{\circ}\right.$, or $\left.45^{\circ}\right)$, were conducted on accuracy (proportion correct), sensitivity $\left(d^{\prime}\right)$, and RT. RT data analysis was conducted using accurate responses.

The $3 \times 4$ ANOVA for accuracy data showed significant main effects of change type $[F(2,56)=16.45, p<.01]$ and orientation $[F(3,84)=7.93, p<.01]$ but no significant interaction between factors $[F(6,168)=1.21, p=.31]$. Figure 5 suggests that a decrease in accuracy occurred as orientation change increased. A trend analysis conducted for the main effect of orientation on accuracy revealed that the linear and quadratic contrasts were significant (both $p$ s $<.05$ ). This pattern was likely due to the greater accuracy at $15^{\circ}$ rotation relative to both $0^{\circ}$ and $30^{\circ}$ rotation.

Even though the interaction between orientation and change type was not significant, Figure 5 shows that the trend at $0^{\circ}$ is similar to that found in Keane et al.'s (2003) results. Indeed, a planned comparison between categorical and coordinate changes at $0^{\circ}$ (mean proportions correct .90 and .83 , respectively) showed a significant difference $(p<.05)$. The same comparison at $45^{\circ}$ (mean proportions correct .85 and .82 , respectively) was not significant ( $p=$ .38). Bonferroni-adjusted post hoc contrasts showed that, when collapsed across orientation, accuracy for shape change was significantly worse than for any other type of change (all $p \mathrm{~s}<.001$; see Figure 5). Although overall detection accuracy for categorical relation changes was greater than for coordinate relation changes (mean pro- 


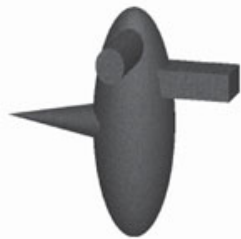

Standard Object

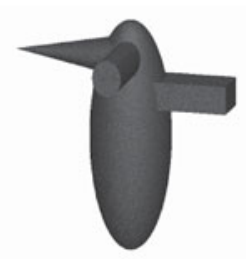

Categorical Change

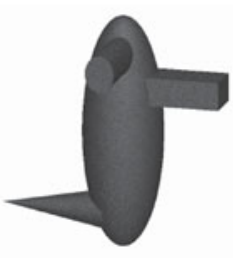

Coordinate Change

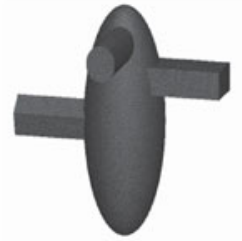

Shape Change

Figure 4. Example of the three different types of change (categorical, coordinate, and shape) used in Experiment 2. The shape change is the same as that described in Experiment 1. The categorical change in this example involves the left part changing its categorical relationship with (1) the middle part, by moving from below to in line with, and (2) the right part, by moving from below to above. The coordinate change in this example involves the left part changing its coordinate relationship with both the middle and right parts by 100 pixels distance.

portions correct .89 and .85 , respectively), this difference was not significant $(p=.19)$. As seen in Figure 5, the $d^{\prime}$ data show the same pattern as the accuracy data. Both main effects were significant, whereas the interaction was not significant. In addition, a Bonferroni-adjusted post hoc contrast showed that overall sensitivity was not significantly different for categorical and coordinate changes $(p=.22)$. These $d^{\prime}$ results indicate that our experimental manipulations altered subjects' sensitivity to change, rather then their response bias.

A $3 \times 4$ repeated measures ANOVA for RT also showed significant main effects for change type $[F(2,56)=8.08$, $p<.01]$ and orientation $[F(3,84)=5.86, p<.01]$, with no significant interaction between change type and orientation $[F(6,168)=0.88, p=.51$; see Figure 6]. Bonferroniadjusted post hoc contrasts showed that there was no difference in RT between the types of configural changes ( $p=$ .83 ) and that both types of configural changes were detected significantly more quickly than were shape changes (both $p \mathrm{~s}<.01$ ). A trend analysis was conducted for the main effect of orientation on RT. The linear contrast was significant $(p<.05)$, indicating that RT increased as the degree of rotation increased. The quadratic contrast was not significant $(p=.07)$.

In Experiment 2, shape changes were detected significantly more slowly and less accurately than were other change types, regardless of object orientation. Categorical and coordinate configural changes were created using quantitatively equal-sized shifts of parts (presumably creating equal-sized changes in pixel change, contour, color, luminance, etc.). Yet, when viewpoint was held constant, there was clearly a difference in subjects' ability to detect categorical and coordinate changes. There was no interac- tion between change type and orientation, however; across all orientations, the speed and accuracy of both categorical and coordinate change detection remained similar. Although changes to categorical spatial relations appeared to be more accurately detected when the viewpoint remained constant, there was little evidence to support the contention that a general difference exists between categorical and coordinate spatial relations across object rotations. Thus, the present data indicate that configural information in terms of both categorical and coordinate relations provides useful information for object discrimination across changes in viewpoint, an idea compatible with such models as Hummel and Stankiewicz's (1998) MetriCat, which encodes both categorical and coordinate spatial relations.

\section{GENERAL DISCUSSION}

Across all manipulations of object stimuli, detection of configural changes remained superior to that of shape and switch changes. In contrast to Johnston and Hayes (2000), both of our experiments showed that regardless of orientation in depth, configural changes to objects were detected more quickly and more accurately than were part shape or switch changes. In attempting to understand the differences in results between these two studies, three task differences might be important. First, the Johnston and Hayes study used object sets that were constructed of a different number of parts. The set that matched in spatial relations consisted of objects with three or four parts, whereas objects that matched in morphology each had five parts. This resulted in different basic configurations of the objects in each set, making comparison between the two sets difficult (indeed, Johnston \& Hayes never 


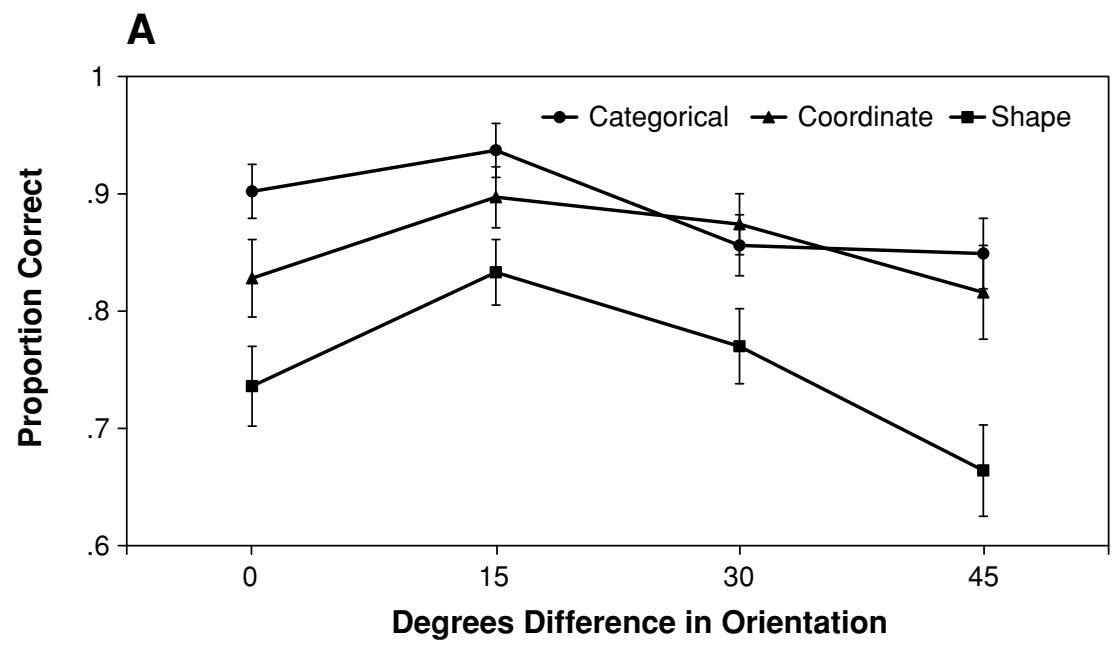

\section{B}

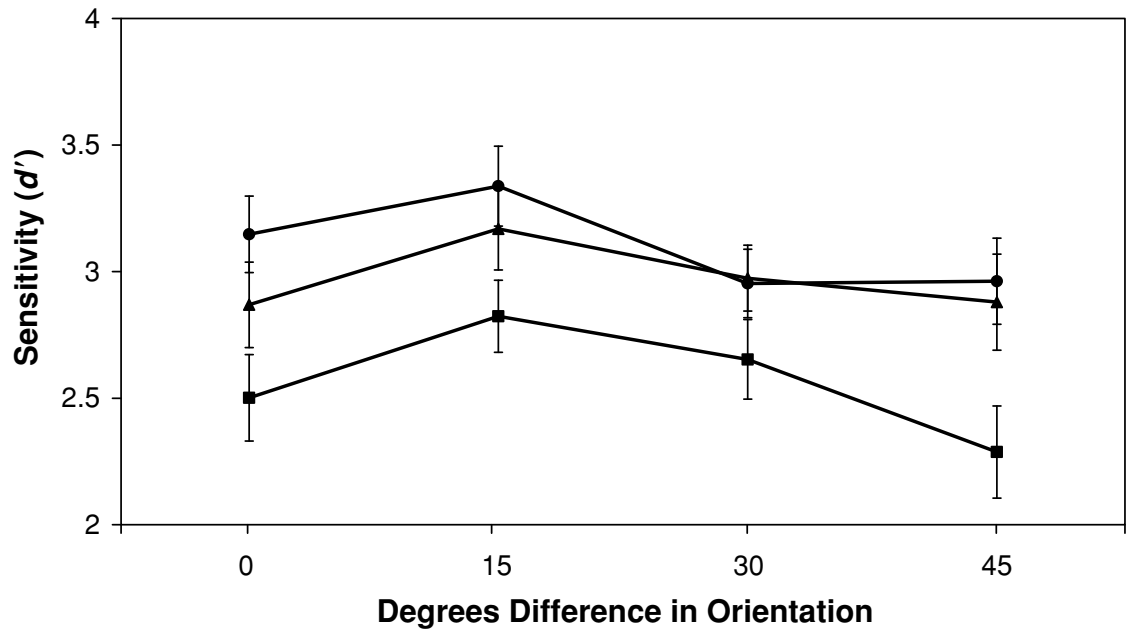

Figure 5. Mean accuracy (A) and sensitivity to change (B) on the change detection task in Experiment 2 as a function of change type and object orientation. Error bars represent $\pm S E$.

made a direct comparison of recognition performance for the two object sets). The increased complexity (number of parts) of the objects discriminable by spatial relations may account, at least partially, for the relatively poorer performance Johnston and Hayes found for this condition. Second, the range of viewpoints used in the Johnston and Hayes study was much larger (up to $180^{\circ}$ ) than the range we used in our study (up to $45^{\circ}$ ).

Third, and perhaps most important, the changes to objects in the Johnston and Hayes (2000) study involved all components - that is, morphology changes altered the shapes of all parts, and changes to spatial relations altered the connectivity of all components. Thus, the subjects in their study performed trials with a greater degree of change in object morphology, configuration, and rotation size. Further, since in the morphology change experiments successful matching and recognition could be done on the basis of just one part (any part), it may not be surprising that performance was better than that in spatial relation change experiments, in which the relations be- tween all parts had to be encoded for successful matching and recognition. By contrast, in the present study we used object stimuli that consisted of the same number of parts (each object had four parts) and also conditions in which changes to part morphology (shape) and spatial relations each involved only one part. The use of stimuli of this kind allowed us to more directly compare spatial relations between parts with part shape. Furthermore, it required subjects to encode objects as a whole at study, since which part would change - or how - was not known. Perhaps a more holistic or global encoding of objects leads to changes of a more global nature (e.g., configuration changes) being easier to detect than local changes (e.g., the shape of one part). Such a hypothesis would need to be investigated in future studies.

In Experiment 2, we demonstrated that the advantage found for changes to spatial relations across object rotation did not depend upon whether the configural change was categorical or coordinate in nature. Changes of similar magnitude to the configuration of an object appeared 


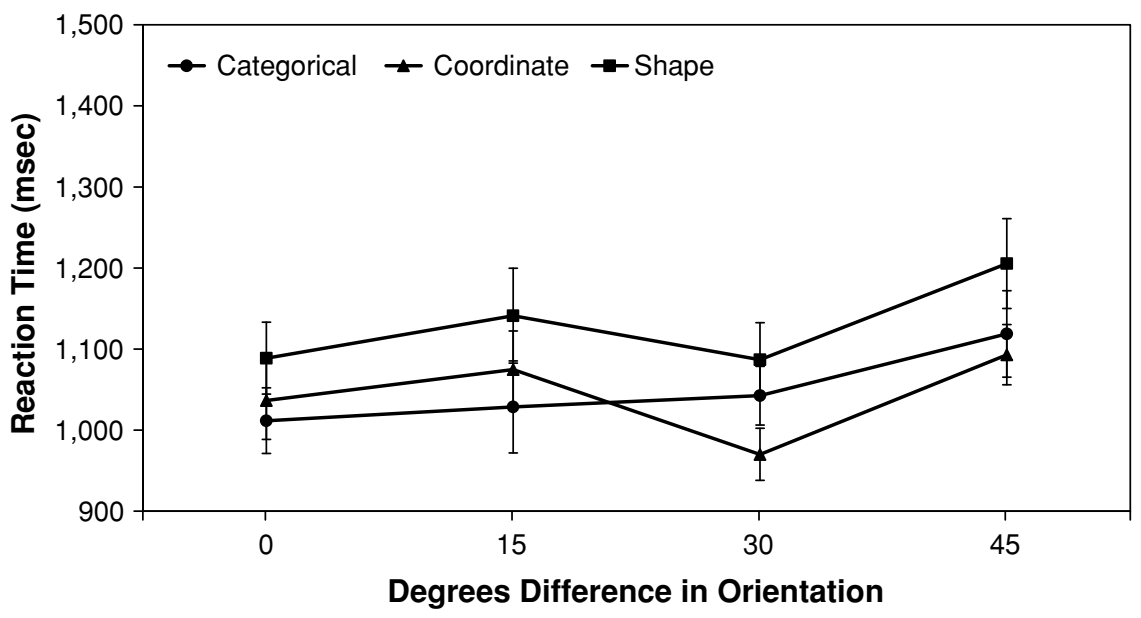

Figure 6. Mean RT on the change detection task in Experiment 2 as a function of change type and object orientation. Error bars represent $\pm S E$.

to be detected at a similar speed and accuracy. The finding of a null result makes it difficult to draw firm conclusions; however, it is interesting to compare these results with those of Hummel and Stankiewicz (1996). In their study, they compared recognition at a single viewpoint of objects that differed in categorical or coordinate spatial relations. Similar to the present results and those of Keane et al. (2003), when viewpoint was fixed, Hummel and Stankiewicz found that subjects were less likely to confuse objects sharing categorical relations than objects sharing coordinate relations. Of interest is whether such differences would remain if they had examined performance across changes in viewpoint. The results of Experiment 2 suggest that this is an important condition to consider.

The data from the present study suggest that coarse configural information - in terms of both categorical and coordinate relations - has a key role in the perception of novel objects across rotation in depth (at least up to rotations of $45^{\circ}$ ), and that the shape of specific components has a reduced role. On the whole, these results provide support for the general idea that structural information may be encoded along with multiple viewpoint-specific representations of objects (Tarr \& Bülthoff, 1998). More specifically, the present findings, together with those of Keane et al. (2003), are compatible with Edelman and Intrator's $(2000,2001,2003) \mathrm{CoF}$ theory of object representation, in which object structure is the principal element of object representation and the parts of an object are coarsely coded shape fragments. In fact, we found that configural changes, and not shape changes, were detected accurately and quickly, regardless of the object's orientation in depth. Furthermore, viewpoint-dependent performance was found across all change types, and configural changes (both coordinate and categorical) were consistently detected more quickly and accurately.

Important to note, however, is that although coarse configural information is used quickly and accurately across different object viewpoints, it appears to be subject to the same decay across changes in viewpoint as other shape information. That is, configural information appears to be qualitatively similar to other kinds of shape information; it simply gives a stronger quantitative signal at all viewpoints. This is compatible with Foster and Gilson's (2002) model of object recognition that combined both structural and view-based information in a simple additive model that included independent terms for object structure and $2-\mathrm{D}$ view information. The Foster and Gilson model accounts for the present results in that it is sensitive to structural features but generally viewpoint dependent.

\section{REFERENCES}

BIEDERMAN, I. (1987). Recognition-by-components: A theory of human image understanding. Psychological Review, 94, 115-147.

Bülthoff, H. H., Edelman, S. Y., \& TarR, M. J. (1995). How are three-dimensional objects represented in the brain? Cerebral Cortex, $\mathbf{5}, 247-260$

Edelman, S., \& Intrator, N. (2000). (Coarse coding of shape fragments $)+($ retinotopy $)=$ Representation of structure. Spatial Vision, 13, 255-264.

Edelman, S., \& Intrator, N. (2001). A productive, systematic framework for the representation of visual structure. In T. K. Leen, T. G. Dietterich, \& V. Tresp (Eds.), Advances in neural information processing systems 13 (pp. 10-16). Cambridge, MA: MIT Press.

EDElman, S., \& Intrator, N. (2003). Towards structural systematicity in distributed, statically bound visual representations. Cognitive Science, 27, 73-109.

Favelle, S. K., Palmisano, S., Burke, D., \& Hayward, W. G. (2006). The role of attention in processing configural and shape information in 3-D novel objects. Visual Cognition, 13, 623-642.

Foster, D. H., \& Gilson, S. J. (2002). Recognizing novel threedimensional objects by summing signals from parts and views. Proceedings of the Royal Society of London: Series B, 269, 1939-1947.

HAYWARD, W. G., \& TARR, M. J. (1997). Testing conditions for viewpoint invariance in object recognition. Journal of Experimental Psychology: Human Perception \& Performance, 23, 1511-1521.

Hummel, J. E., \& Stankiewicz, B. J. (1996). Categorical relations in shape perception. Spatial Vision, 10, 201-236.

Hummel, J. E., \& Stankiewicz, B. J. (1998). Two roles for attention in shape perception: A structural description model of visual scrutiny. Visual Cognition, 5, 49-79.

Johnston, M. B., \& HaYes, A. (2000). An experimental comparison of viewpoint-specific and viewpoint-independent models of object 
representation. Quarterly Journal of Experimental Psychology, 53A, $792-824$

Keane, S. K., Hayward, W. G., \& Burke, D. (2003). Detection of three types of changes to novel objects. Visual Cognition, 10, 101-127.

KIMCHI, R. (2003). Relative dominance of holistic and component properties in the perceptual organization of visual objects. In M. A. Peterson \& G. Rhodes (Eds.), Perception of faces, objects, and scenes. Analytic and holistic processes. Oxford: Oxford University Press.

Macmillan, N. A., \& Creelman, C. D. (1991). Detection theory: A user's guide. New York: Cambridge University Press.

TARR, M. J., \& BüLTHOFF, H. H. (1998). Image-based object recognition in man, monkey and machine. Cognition, 67, 1-20.

Tarr, M. J., Bülthoff, H. H., Zabinski, M., \& Blanz, V. (1997). To what extent do unique parts influence recognition across changes in viewpoint? Psychological Science, 8, 282-289.

Tarr, M. J., Williams, P., Hayward, W. G., \& Gauthier, I. (1998).
Three-dimensional object recognition is viewpoint dependent. Nature Neuroscience, 1, 275-277.

Williams, P., \& Simons, D. J. (2000). Detecting changes in novel, complex three-dimensional objects. Visual Cognition, 7, 297-322.

\section{NOTES}

1. These are in terms of the color values, so the maximum difference was obtained by changing a purely black image to a purely white image (this change scored 100\%; any other change scored less than $100 \%$ ).

2. Given that all components had the same physical color (purple), this measure reflects increasing differences in color caused by-for example - increasing rotations of an object that systematically change surface angle with respect to lighting and therefore make a surface seem brighter or darker.

\section{APPENDIX}

\begin{tabular}{|c|c|c|c|c|c|c|c|c|}
\hline \multicolumn{9}{|c|}{$\begin{array}{c}\text { Table A1 } \\
\text { Mean Values for Percentage Pixel Change From "Standard" } \\
\text { Object in Experiment } 1 \text { As a Function of Change Type (Including Same) } \\
\text { and Object Orientation }\end{array}$} \\
\hline \multirow{2}{*}{$\begin{array}{c}\text { Degrees } \\
\text { Difference in } \\
\text { Orientation }\end{array}$} & \multicolumn{2}{|c|}{ Same } & \multicolumn{2}{|c|}{ Configuration } & \multicolumn{2}{|c|}{ Switch } & \multicolumn{2}{|c|}{ Shape } \\
\hline & $M$ & $\overline{S E}$ & $M$ & $S E$ & $M$ & $S E$ & $M$ & $S E$ \\
\hline 0 & 0 & 0 & 3.66 & 0.22 & 2.90 & 0.54 & 1.40 & 0.19 \\
\hline 15 & 3.33 & 0.60 & 5.78 & 0.60 & 4.82 & 0.87 & 4.07 & 0.64 \\
\hline 30 & 6.12 & 0.78 & 7.45 & 0.83 & 6.59 & 1.04 & 6.41 & 0.88 \\
\hline 45 & 7.76 & 0.93 & 8.11 & 0.94 & 7.83 & 1.11 & 7.81 & 1.01 \\
\hline
\end{tabular}

Table A2

Mean Values for Percentage Color Change From "Standard" Object in Experiment 1 As a Function of Change Type (Including Same) and Object Orientation

\begin{tabular}{|c|c|c|c|c|c|c|c|c|}
\hline \multirow{2}{*}{$\begin{array}{c}\text { Degrees } \\
\text { Difference in } \\
\text { Orientation }\end{array}$} & \multicolumn{2}{|c|}{ Same } & \multicolumn{2}{|c|}{ Configuration } & \multicolumn{2}{|c|}{ Switch } & \multicolumn{2}{|c|}{ Shape } \\
\hline & $M$ & $S E$ & $M$ & $S E$ & $M$ & $S E$ & $M$ & $S E$ \\
\hline 0 & 0 & 0 & 3.01 & 0.24 & 2.83 & 0.52 & 1.64 & 0.30 \\
\hline 15 & 3.35 & 0.50 & 4.98 & 0.48 & 4.43 & 0.68 & 3.89 & 0.54 \\
\hline 30 & 5.28 & 0.60 & 6.14 & 0.63 & 5.64 & 0.77 & 5.52 & 0.69 \\
\hline 45 & 6.33 & 0.74 & 6.58 & 0.74 & 6.43 & 0.82 & 6.43 & 0.80 \\
\hline
\end{tabular}

(Manuscript received November 2, 2004;

revision accepted for publication December 7, 2005.) 